\title{
Frailty Syndrome Prevalence and Its Correlation with Cognitive Function in Elderly Surgical Inpatients
}

\author{
Feifan Liu, Yanlin Heng*, Qianwen Li, Qingyi He \\ Department of Medicine, Yangtze University, Jingzhou, China \\ Email:`397515757@qq.com
}

How to cite this paper: Liu, F.F., Heng, Y.L., Li, Q.W. and He, Q.Y. (2020) Frailty Syndrome Prevalence and Its Correlation with Cognitive Function in Elderly Surgical Inpatients. Journal of Biosciences and Medicines, 8, 148-156.

https://doi.org/10.4236/jbm.2020.810013

Received: August 11, 2020

Accepted: October 19, 2020

Published: October 22, 2020

Copyright $\odot 2020$ by author(s) and Scientific Research Publishing Inc. This work is licensed under the Creative Commons Attribution-NonCommercial International License (CC BY-NC 4.0). http://creativecommons.org/licenses/by-nc/4.0/

\begin{abstract}
Background: Frailty is a geriatric syndrome, and a common negative consequence of aging, which shares some obvious characteristics as cognitive impairment. Preventing and relieving frailty may reduce the possibility of developing cognitive impairment. Objective: This study aimed to investigate frailty prevalence and its correlation with cognitive function in elderly surgical inpatients. Methods: We enrolled a random sample of elderly surgical inpatients from Jingzhou from June 2020 to August 2020. We used a self-made registration sheet to collect their demographic data (gender, age, nationality, educational level and monthly income), and used the FRAIL Scale to assess the prevalence of frailty, and used the Mini-Mental State Examination (MMSE) to assess cognitive function. We used multiple linear regression analysis to explore the correlation between frailty level and demographic data, then Pearson correlation analysis was performed to analyze the correlation between frailty and cognitive function. Results: In the 143 elderly surgical inpatients, prevalence of frail was seen in 29 (20.3\%), and pre-frail state was found in $64(44.8 \%)$. Only 50 (34.9\%) were in healthy state. Cognitive impairment was seen in 28 (19.5\%). Pearson correlation analysis showed that the frail scoring was correlated with cognitive function in elderly surgical inpatients $(\mathrm{r}=-0.378, \mathrm{P}<0.05)$. Conclusion: The prevalence of frailty is high in elderly surgical inpatients, which is closely related to age and gender. Elderly surgical inpatients have high cognitive impairment, and frailty appears strongly associated with cognitive status. The findings suggest that attention should be paid to the frailty and cognitive function in the elderly surgical inpatients by pro-vision of effective interventions.
\end{abstract}

\section{Keywords}

Frailty Syndrome, Cognitive Impairment, Aged, Surgical Inpatients, Mini Mental State Examination 


\section{Introduction}

Frailty syndrome is an important geriatric syndrome, which refers to the elderly's decreased reserve function of multiple physiological systems, increased vulnerability, decreased ability to maintain homeostasis and decreased ability to resist stress, and can also lead to a series of adverse consequences in the face of small stimuli [1], the occurrence of which is closely related to various adverse outcomes such as falls, unplanned hospitalization, prolonged hospitalization, reduced quality of life, and death [2]. Foreign studies have shown that frailty syndrome is closely related to cognitive function [3]. Cognitive function is the higher function of the brain, which refers to human's comprehensive perception of external things [4]. By the end of 2020, the number of people over the age of 60 is expected to increase to 255 million, accounting for $17.8 \%$ of the total population [5]. Early identification and management of geriatric surgery patients can not only improve the quality of life of patients, but also reduce the financial burden of caregivers. At present, there are few studies on the correlation between frailty syndrome and cognitive function in elderly surgical inpatients in China. By discussing the current situation and correlation between frailty syndrome and cognitive function in surgical inpatients, this study provides some theoretical basis for the intervention of the progression of frailty syndrome and cognitive function decline.

\section{Research Object}

This study was conducted using convenience sampling method with 143 elderly surgical inpatients from June 2020 to August 2020 in the departments of gastrointestinal surgery, orthopedics, and cardiothoracic surgery at Jingzhou first people's Hospital. Inclusion criteria: age $>60$ years; volunteer to participate in this study and sign informed consent before the start. Exclusion criteria: severe diseases and terminal diseases; authors of acute or chronic diseases; taking carbidopa/Levodopa, Donepezil hydrochloride, and antidepressants (which can produce debilitating symptoms); unable to cooperate with the completion of the assessment due to disease or other reasons.

\section{Research Methods}

\subsection{Research Tools}

\subsubsection{The General Information Questionnaire}

The general information questionnaire was compiled by the investigator, including gender, age, education, residence, marital status, comorbidities, multiple drug use, etc.

\subsubsection{FRAIL Scale}

The FRAIL Scale, is a clinical frailty screening tool for the elderly put forward by an international expert group on nutrition, health and aging in 2008 [6]. The questionnaire form, a total of five items scale: fatigue (in the past 1 month feel- 
ing fatigue), endurance (independent and 10 steps difficulty), line power limited (walk $100 \mathrm{~m}$ ), sickly coexistence (more than 5 kinds of chronic disease) and weight (weight loss in recent one year more than 5\%), each 1 minute, 1 - 2 points hint at the beginning of the weak, have to tip more than three points [7]. The structure validity and content validity of the scale were both good, and the area under ROC curve was 0.71 , indicating that the test accuracy of the scale was high. The reliability and validity of the scale was good, with a Cronbach's coefficient of 0.825 , suggesting that it was suitable for the evaluation of hospitalized elderly patients with frailty.

\subsubsection{Mini-Mental State Examination (MMSE)}

This tool is a simple tool for the classification of cognitive impairment in the elderly. It consists of five parts, namely, orientation, memory (instantaneous and short-term memory), attention and computation, aphasia (oral expression, naming, retelling, reading, listening comprehension, and writing) and vision, with a total of 30 points. The cognitive status of the elderly was evaluated by combining their educational level and cognitive score. Assessment criteria for cognitive impairment in the elderly [8] [9]: According to the level of education, MMSE score $\leq 19$, primary school $\leq 22$, junior high school and above $\leq 26$ is classified as cognitive impairment.

\subsection{Data Collection}

In this study, relevant data were collected by sending questionnaires to discharged elderly surgical inpatients who met the inclusion criteria. 150 questionnaires were issued and 143 effective questionnaires were recovered, the effective recovery rate was $95.33 \%$.

In the process of this study, the researcher explained to the respondents the purpose and significance of the study and the matters needing attention in filling the questionnaire. After obtaining their informed consent, the researcher used the unified guidance language to evaluate the respondents. Geriatric surgery patients with primary school education or below were asked to state the questionnaire items one by one, and the respondents were asked to choose their own options. Questionnaires were handed out on the spot and collected on the spot.

\subsection{Statistical Analysis}

SPSS 20.0 software was used for statistical analysis. The general data and the scores of each scale were described by Mean \pm SD, frequency, and percentage. Two independent sample t-tests were used for comparison between groups, and the comparison between groups was $\mathrm{x}^{2}$ Check analysis. Logistic regression model was used to analyze the influencing factors of frailty syndrome. Multiple linear regression analysis and Pearson correlation analysis were used to explore the correlation between cognitive function and frailty syndrome in geriatric surgery inpatients. The significance of the test was at $=0.05$ level, and $\mathrm{P}<0.05$ was considered statistically significant. 


\section{Results}

\subsection{Comparison of Frailty Syndrome Scores among Patients with Different Demographic Characteristics}

A total of 143 patients were included in this study, including 84 males (58.7\%) and 59 females $(41.3 \%)$. The average age was $(71.24 \pm 6.8)$ years old. While on the other hand, according to the score of the geriatric scale (0 as non-frail, 1 - 2 as early FRAIL, $\geq 3$ as FRAIL), the elderly surgical inpatients were grouped, of which $50(34.9 \%)$ were in the non-frail group, $64(44.8 \%)$ in the per-frail group, and $29(20.3 \%)$ in the frail group.

\subsection{Single Factor Analysis of Frailty Syndrome in Elderly Surgical Patients}

Statistical analysis was performed on 143 subjects who met the inclusion and exclusion criteria. The differences in age, sex, body mass index, type of chronic diseases and amount of drugs taken between the frailty group and the non-frailty group were statistically significant $(\mathrm{P}<0.05)$. There were no statistically significant differences in the incidence of frailty syndrome among different ethnic groups, education level, religious belief, personal monthly income, smoking and drinking ( $\mathrm{P}>0.05)$. The results were shown in Table 1 .

Table 1. Comparison of frailty status of geriatric surgical inpatients with different general data $(n=143)$

\begin{tabular}{|c|c|c|c|c|c|c|}
\hline \multirow{2}{*}{ Characteristics } & \multirow{2}{*}{$\begin{array}{c}\text { Case } \\
(\%)\end{array}$} & \multicolumn{3}{|c|}{ Frailty baseline survey } & \multirow{2}{*}{$\begin{array}{c}\text { Statistic } \\
\text { value }\end{array}$} & \multirow{2}{*}{ P-value } \\
\hline & & Non-frail & Per-frail & Frail & & \\
\hline \multicolumn{7}{|l|}{ Nation } \\
\hline Han nationality & $139(97.2)$ & $34(94.4)$ & $63(98.4)$ & $42(97.7)$ & $1.105^{1)}$ & 0.575 \\
\hline Ethnic minority & $4(2.8)$ & $2(5.6)$ & $1(1.6)$ & $1(2.3)$ & & \\
\hline \multicolumn{7}{|l|}{ The Age (y) } \\
\hline $60-65$ & $37(25.9)$ & $17(47.2)$ & $17(26.6)$ & $3(7.0)$ & $25.819^{1)}$ & 0.004 \\
\hline $66-70$ & $36(25.2)$ & $9(25.0)$ & $18(28.1)$ & $9(20.9)$ & & \\
\hline $71-75$ & $22(15.4)$ & $4(11.1)$ & $11(17.2)$ & $7(16.3)$ & & \\
\hline $76-80$ & $37(25.9)$ & $6(16.7)$ & $13(20.3)$ & $18(41.9)$ & & \\
\hline $81-85$ & $10(7.0)$ & 0 & $5(7.8)$ & $5(11.6)$ & & \\
\hline $86-90$ & $1(0.7)$ & 0 & 0 & $1(2.3)$ & & \\
\hline \multicolumn{7}{|l|}{ Gender } \\
\hline Male & $84(58.7)$ & $25(69.4)$ & $37(57.8)$ & $22(51.2)$ & $7.477^{1)}$ & 0.024 \\
\hline female & $59(41.3)$ & $11(30.6)$ & $27(42.2)$ & $21(48.8)$ & & \\
\hline \multicolumn{7}{|l|}{ Education level } \\
\hline Illiteracy & $33(28.7)$ & $7(18.9)$ & $17(27.0)$ & $9(20.9)$ & $3.421^{1)}$ & 0.970 \\
\hline Primary school & $39(29.3)$ & $9(24.3)$ & $15(23.8)$ & $15(34.9)$ & & \\
\hline
\end{tabular}




\section{Continued}

\begin{tabular}{|c|c|c|c|c|c|c|}
\hline Junior middle school & $35(24.5)$ & $8(21.6)$ & $17(27.0)$ & $10(23.3)$ & & \\
\hline High school & $33(23.1)$ & $11(29.7)$ & $13(20.6)$ & $9(20.9)$ & & \\
\hline College & $3(2.1)$ & $2(5.4)$ & $1(1.4)$ & 0 & & \\
\hline \multicolumn{7}{|l|}{ Religious are } \\
\hline Yes & $10(6.9)$ & $2(5.6)$ & $5(7.8)$ & $3(7)$ & $0.581^{1)}$ & 0.965 \\
\hline NO & $133(93.1)$ & $34(94.4)$ & $59(92.2)$ & 40 (93). & & \\
\hline \multicolumn{7}{|c|}{ Fmilay per Capita Monthly Ibcome (RMB) } \\
\hline$<3000$ & $89(62.2)$ & $21(55.3)$ & $32(60.4)$ & $36(69.2)$ & $8.824^{1)}$ & 0.184 \\
\hline $3000-5000$. & $32(22.4)$ & $8(21.1)$ & $14(26.4)$ & $10(19.2)$ & & \\
\hline $5000-8000$. & $20(14.0)$ & $8(21.1$ & $6(11.3)$ & $6(11.6)$ & & \\
\hline$>8000$ & $2(1.4)$ & $1(2.5)$ & $1(1.9)$ & 0 & & \\
\hline \multicolumn{7}{|l|}{ Smoking } \\
\hline No smoking & $79(55.3)$ & $30(58.8)$ & $41(51.9)$ & $8(72.7)$ & $7.038^{1)}$ & 0.134 \\
\hline The Quit smoking & $37(27.6)$ & $9(17.0)$ & $27(34.2)$ & $1(9.1)$ & & \\
\hline The Frequent smoking & $27(20.1)$ & $14(23.5)$ & $11(13.9)$ & $2(18.2)$ & & \\
\hline \multicolumn{7}{|l|}{ Drinking } \\
\hline No drinking & $79(55.3)$ & $30(56.6)$ & $41(51.9)$ & $8(72.7)$ & $3.093^{1)}$ & 0.542 \\
\hline The Quit smoking & $28(19.6)$ & $8(15.1)$ & $18(22.8)$ & $2(18.2)$ & & \\
\hline The Frequent smoking & $36(25.1)$ & $15(28.3)$ & $20(25.3)$ & $1(9.1)$ & & \\
\hline BMI & $21.97+/-2.81$ & $22.50+/-2.63$ & $21.93+/-2.80$ & $19.75+/-2.89$ & $4.571^{2)}$ & 0.012 \\
\hline \multicolumn{7}{|l|}{ Complication } \\
\hline None & $76(53.1)$ & $26(71.4)$ & $36(56.3)$ & $15(34.9)$ & 4 & 0.047 \\
\hline $1-3$ & $56(39.2)$ & $8(22.9)$ & $27(42.2)$ & $21(48.8)$ & & \\
\hline$>3$ & $10(7.7)$ & $2(5.7)$ & $1(1.6)$ & $7(16.3)$ & & \\
\hline \multicolumn{7}{|c|}{ The Number of medications seems } \\
\hline 0 & $61(42.7)$ & $21(39.6)$ & $38(48.1)$ & 2 & $13.766^{1)}$ & 0.008 \\
\hline $1-4$ & $66(46.1)$ & $29(54.7)$ & $31(39.2)$ & 6 & & \\
\hline 5 or more & $16(11.1)$ & $3(5.7)$ & $10(12.6)$ & 3 & & \\
\hline
\end{tabular}

${ }^{1)} \mathrm{X}^{2}$-test, ${ }^{2)} \mathrm{F}$-test.

\subsection{Results of Linear Regression Analysis on Influencing Factors of Frailty Syndrome in Elderly Surgical Patients}

FRAIL scale score as dependent variable based on age (60 - 65 years old $=1 ; 66$ 70 years $=2 ; 71-75$ years $=3 ; 76-80$ years $=4 ; 81-85$ years $=5 ; 86-90$ years old $=6$ ), gender $($ male $=1$; female $=2)$, BMI, type of chronic disease (none $=1 ; 1$ - $3=2$; 3 types of $\geq 3$ ), types of drugs currently in use (none $=1 ; 1-4=2$; Multiple linear regression analysis with $\geq 3$ as independent variable. Showed that age, gender and type of drugs currently used were the influential factors in the FRAIL scale score $(\mathrm{P}<0.05)$. The results were shown in Table 2. 
Table 2. Multiple linear regression analysis of the factors influencing the score of elderly inpatients with surgery scale.

\begin{tabular}{cccccc}
\hline Variable & $B$ & $S E$ & Standardized B & $t$ & $\mathrm{P}$ \\
\hline Constant & 0.515 & 0.760 & & 0.678 & 0.000 \\
Gender & 0.429 & 0.170 & 0.168 & 2.519 & 0.013 \\
Age & 0.243 & 0.067 & 0.253 & 3.634 & 0.001 \\
BMI & 0.045 & 0.035 & 1.00 & 1.277 & 0.204 \\
Type of chronic diseases & 0.208 & 0.125 & 0.112 & 1.659 & 0.099 \\
Types of drugs currently in use & 0.713 & 0.125 & 0.399 & 5.702 & 0.003 \\
\hline $\mathrm{R}^{2}=0.376, \mathrm{~F}=20.813, \mathrm{P}<0.001$. & & & & &
\end{tabular}

\subsection{Correlation Analysis of Cognitive Function Status and Frailty Status in Geriatric Surgery Inpatients}

143 elderly cases of hospitalized patients with surgical MMSE score was (23.86 \pm 3.42), the prevalence of cognitive impairment was $19.5 \%$, the cognitive function status as shown in Table 3. Using Pearson correlation analysis: surgical geriatric inpatients MMSE scores and FRAIL scale score of correlation coefficient is $-0.378(\mathrm{P}=0.000<0.05)$, suggesting a surgical hospitalized elderly patients decline with the increase of the degree of weak MMSE score, the difference is statistically significant.

\section{Discussion}

In this study, the incidence of frailty syndrome in geriatric surgery inpatients was $20.3 \%$, and the incidence of prefrailty was $44.8 \%$. Currently, there are few studies on the prevalence of frailty syndrome in geriatric surgery inpatients in China. Based on Qiuping Li et al. [10] showed that the incidence of frailty syndrome in geriatric surgery inpatients was $23.88 \%$. The incidence of frailty syndrome in inpatients was reported to be $26.6 \%$ - 58.9\% [11] [12] [13] [14]. Overall, it was slightly higher than that in this study, which may be related to the selection of subjects in this study. Inpatients with acute onset and short course of disease in surgery had less stress effect compared with patients with other medical diseases. At the same time, ethnicity, condition, assessment tools for frailty syndrome, and sample size also have a certain influence on the incidence of frailty syndrome. Reversibility is one of the characteristics of frailty syndrome [15], the geriatric surgery inpatients should be evaluated for frailty syndrome, early detection and recognition, and appropriate treatment, part of the frailty state can be reversed to the early frailty, and the early frailty can be reversed to the normal state [16]. So as to reduce incapacity, fall and other adverse outcomes, reduce hospitalization rate, mortality rate [17].

The results showed that frailty was associated with age and sex, as well as with the type of drug used. The results suggests that older, more female patients and those taking more medications are at greater risk for frailty syndrome. 1) There is a close relationship between age and frailty, and the increase of age is usually 
Table 3. Cognitive function of elderly surgical inpatients with different frailty conditions.

\begin{tabular}{ccc}
\hline Group & Cognitive impairment & Cognitively normal \\
\hline Non-frail & 10 & 43 \\
Per-frail & 16 & 48 \\
Frail & 8 & 18 \\
\hline
\end{tabular}

associated with different degrees of frailty. Previous studies have suggested that the incidence of frailty increases with age [18]. This is consistent with the results of this study. With the increase of age, each organ of the patient's body gradually tends to be weak, and each physiological function gradually decreases, and the stress function becomes worse. Even a small stimulus may cause the disorder of balance in the patient's body, leading to the occurrence of weakness [19]. This suggests the importance of early frailty synthesis in screening. 2) The incidence of frailty in female is higher than that in male [20]. This study shows that female is a risk factor for frailty in elderly hospitalized fracture patients, consistent with previous findings. Female is more prone to frailty than male, which may be related to thinner women, weaker muscle mass and strength [21]. 3) The type of drugs used is an influential factor in the state of frailty, which is consistent with the results reported in the literature [22]. In view of the co-existence of multiple diseases in the elderly, there are many kinds of drugs, but it is not possible to exclude the weakness caused or aggravated by the interaction between drugs due to the excessive variety of drugs applied. The relationship between the two is not clear at present, and further prospective studies are needed to explore. Therefore, the management of multiple drug use should be done, rational drug use should be done and improper drug use should be corrected in time.

This study showed that the incidence of cognitive impairment in geriatric surgery inpatients was $20.3 \%$, and the total score of weak MMSE was negatively correlated with the total score of MMSE $(\mathrm{R}=-0.378, \mathrm{P}<0.05)$, that is, the elderly patients with frailty and cognitive decline, which was consistent with the results of a number of foreign studies [3]. The main reasons are as follows: 1) Frailty and cognitive function are closely related to chronic inflammation, pathophysiology, hormone imbalance, nutritional status, vascular disease risk, mental and psychological health, etc. Therefore, the elderly with early frailty or frailty are also accompanied by the decline of cognitive function [23]; 2) In the elderly with frailty, due to the decline of physical activity ability, slow walking pace, fatigue and other reasons, the ability of exercise and exercise is reduced, leading to the corresponding reduction of the elderly's exposure to external information stimulation, and thus the decline in cognitive function and further cognitive impairment. There are complex mechanisms between debilitation and cognitive function that are difficult to explain by common biological pathways, but the development of both is closely related to poor nutritional status, inflammatory markers, and chronic diseases (diabetes, cardiovascular disease, and chronic obstructive pulmonary disease) [24], and there are interactions between 
the various influences and the mechanisms that exist to weaken organismal function and cognition. Therefore, in daily nursing work, high attention should be paid to the cognitive function of elderly inpatients, especially those with frailty or early frailty, and the elderly with cognitive dysfunction should be recognized as early as possible.

\section{Conclusion}

The prevalence of frailty syndrome and cognitive impairment was higher in geriatric surgery inpatients, and age, cognition, gender and type of drug used were the main influencing factors of frailty syndrome. Therefore, nursing staff should pay attention to the frailty screening of elderly patients hospitalized in surgery, and strengthen the management of cognitive function of elderly patients, and actively seek related reasons for the existence of frailty and cognitive disorder, to improve and remove the reasons. It can improve frailty and cognitive function through exercise and diet, prevent, delay and even reverse frailty and cognitive function decline. The sample size of this study is relatively low, and the mechanism of the interaction between frailty and cognition needs further study.

\section{Conflicts of Interest}

The authors declare no conflicts of interest regarding the publication of this paper.

\section{References}

[1] Fried, L.P., Tangen, C.M., Walston, J., et al. (2001) Frailty in Older Adults: Evidence for a Phenotype. Journals of Gerontology. Series A, Biological Sciences and Medical Sciences, 56, M146-M156. https://doi.org/10.1093/gerona/56.3.M146

[2] Fried, L.P., Ferrucci, L., Darer, J., et al. (2004) Untangling the Concepts of Disability, Frailty, and Comorbty Desert for Improved Targeting and Care. The Journals of Gerontology, Series A: Biological Sciences, 59, 255-263. https://doi.org/10.1093/gerona/59.3.M255

[3] Armstrong, J., Godin, J., Launer, L.J., et al. (2016) Changes in Frailty Predict Changes in Cognition in Older Men: The Honolulu-Asia Aging Study. Journal of Alzheimer's Disease, 53, 1003-1013. https://doi.org/10.3233/JAD-151172

[4] Wang, S.Y., Qin, Q.-B., Wang, M.-J., et al. (2015) Comparison of MoCA and MMSE in Cognitive Function Assessment of Elderly Patients. Chinese Journal of Medical Innovation, 12, 106-108.

[5] Of the State Council about Print and Distribute "Much Starker Choices- and Graver Consequences-In" National Undertaking Development and Pension System Construction Plan, Notify. http://www.gov.cn/zhengce/content/2017-03/06/content 5173930.htm

[6] Xi, X., Guo, G.F. and Sun, J. (2015) Research Progress of Frailty Assessment Tools and Their Application in the Elderly. Chinese Journal of Gerontology, No. 20, 5993-5996.

[7] Ying, Q.Y., Liu, H.P., Guo, X.Y., et al. (2017) Summary of Evidence for Screening and Assessment of Frailty in the Elderly. Journal of Nursing, 32, 95-98. 
[8] Pagonabarraga, J., Kulisevsky, J., Llebaria, G., et al. (2008) Parkinson's Disease-Cognitive Rating Scale: A New Cognitive Scale Specific for Parkinson's Disease. Movement Disorders, 23, 998-1005. https://doi.org/10.1002/mds.22007

[9] Dalrymple-Alford, J.C., Macaskill, M.R., Nakas, C.T., et al. (2010) The MoCA: Well Photographed Screen for Cognitive Impairment in Parkinson Disease. Neurology, 75, 1717-1725. https://doi.org/10.1212/WNL.0b013e3181fc29c9

[10] Li, Q. and Han, B.R. (2017) Investigation and Analysis of the Current Status and Influencing Factors of Debility in Elderly Surgical Inpatients. Nursing Research, 31, 4307-4311.

[11] Tang, W., Jiang, C.Y., Sun, Y., et al. (2019) Evaluation of Fattening State in Elderly Inpatients. Journal of Clinical and Experimental Medicine, 18, 187-192. (In Chinese)

[12] Xiao, M., Mao, J.L. and Zhang, X.J. (2020) Cross-Sectional Analysis of Weakness Risk Factors for Hospitalized Elderly Patients. China General Clinical, 36, 353-358.

[13] Wang, N., Qu, H. and Wang, L. (2020) Analysis of Frailty Status and Influencing Factors of Hospitalized Elderly Patients. Qilu Nursing Journal, 26, 74-77.

[14] Yang, Y.R., Wang, Z.Q., Li, Z., et al. (2020) Analysis of Frailty Status and Its Correlation with Cognitive Function in Hospitalized Patients over 60 Years Old. Chinese Journal of General Practice, 23, 2132-2136. (In Chinese)

[15] Lee, J.S., Auyeung, T.W., Leung, J., et al. (2014) Transitions in Frailty States among Social-Living Older Adults and Their Associated Factors. Journal of the American Medical Directors Association, 15, 281-286. https://doi.org/10.1016/j.jamda.2013.12.002

[16] Yu, P. and Wang, J.Y. (2015) Strengthening Prevention and Treatment of Frailty Syndrome in the Elderly. Chinese Journal of Gerontology, 34, 1281.

[17] Chinese Society of Geriatrics, Chinese Medical Association (2017) Expert Consensus on Frailty Assessment and Intervention in Geriatrics. Chinese Journal of Gerontology, 36, 251-256.

[18] Clegg, A., Young, J., Iliffe, S., et al. (2013) Frailty in Elderly People. The Lancet, 381, 752-762. https://doi.org/10.1016/S0140-6736(12)62167-9

[19] Fu, M.X. and Zhang, X.G. (2019) Research Progress of Frailty Syndrome in the Elderly. Nursing Research, 33, 2973-2976. (In Chinese)

[20] Ning, X.X., Ouyang, M. and Leng, S. (2015) Pathogenesis, Assessment and Management of Frailty Syndrome in the Elderly. Chinese Journal of Geriatrics, 34, 1282-1285.

[21] Cai, K.-Q., Li, W., Wang, L., et al. (2019) Investigation and Study on the Frailty Status of Hemodialysis Patients. Journal of Nursing, 34, 24-27.

[22] Liu, Z.F. and Jian, Z.J. (2015) Frailty: An Important Geriatric Syndrome. Chinese Journal of Gerontology, 34, 1286-1288.

[23] Sun, C., Shi, H., Xi, H., et al. (2018) Frailty and Cognitive Dysfunction. Chinese Journal of Geriatrics, 37, 826-830. (In Chinese)

[24] Pilotto, A., Matera, M.G., Ferrucci, L., et al. (2009) Association of Apolipoprotein E and Angiotensin Converting Enzyme Gene Polymorphisms with the Multidimensional Impairment in Older Patients. Rejuvenation Research, 12, 239-247. https://doi.org/10.1089/rej.2009.0858 